\title{
Direct synthesis of carbon nanotube aerogel using floating catalyst chemical vapor deposition: effect of gas flow rate
}

\begin{abstract}
Carbon nanotube aerogel (CNT aerogel) was synthesized through the catalytic decomposition of toluene in the mixture of ferrocene and thiophene through floating catalyst chemical vapor deposition method. This study described the effect of (Ar and H2) carrier gas flow rate on the quality and yield of CNT aerogel. Results indicated that the flow rate of the gas was an important parameter which helped in determining the yield and quality of the CNTs. The CNTs are multiwalled with its diameter decreased when the $\mathrm{H} 2$ flow rate was increased. Scanning electron microscopy indicated the average diameter of $\approx 31,30,27,26,18$, and $15 \mathrm{~nm}$ when the $\mathrm{H} 2$ flow rate was increased from 400, 450, 500, 550, 600, $650 \mathrm{sccm}$, respectively. Furthermore, elemental analysis with energy dispersion spectrum for CNT aerogel produced at $600 \mathrm{sccm}$ resulted in a $96.22 \mathrm{wt} \%$ carbons along with $3.78 \mathrm{wt} \%$ iron in the CNT aerogel sample. Results indicated that a hydrogen flow rate of $600 \mathrm{sccm}$ produced high yield and good CNTs morphology.
\end{abstract}

